\title{
A New Method for Blocking Probability Evaluation in OBS/OPS Networks With Deflection Routing
}

\author{
Eric W. M. Wong, Senior Member, IEEE, Jayant Baliga, Moshe Zukerman, Fellow, IEEE, Andrew Zalesky, and
} Garvesh Raskutti

\begin{abstract}
In this paper, we present a new method for the estimation of blocking probabilities in bufferless optical burst or packet switched networks. In such networks, deflection routing is used to reduce blocking probability. However, it requires certain wastage due to trunk reservation that must be used to avoid instability. We provide a wide range of simulation and numerical results to validate our new approximation method and demonstrate various effects on blocking probability and utilization, such as network size, trunk size, the maximal number of allowable deflections, and burst/packet length.
\end{abstract}

Index Terms-Blocking probability, deflection routing, Erlang fixed-point approximation, non-hierarchical networks, optical burst switched (OBS), optical packet switching (OPS), overflow priority classification approximation.

\section{INTRODUCTION}

A $S$ internet traffic increases, the capacity of the networks that transport this traffic must also increase. Future all-optical networks are seen as a way to meet this growing demand [1], [2]. However, for all-optical networks to be economically feasible, improved methods of network dimensioning are required. A key component of network dimensioning is the accurate estimation of end-to-end blocking probability in the network [3]-[5]. In this paper, we present a new method for the estimation of end-to-end blocking probabilities in bufferless optical burst switched (OBS) [1] networks considering Just-In-Time (JIT) signalling [6], or optical packet switching (OPS) networks [2], [7]. The concepts of blocking probability, and end-to-end blocking probability, which are used interchangeably, are equivalent to the so-called burst/packet loss ratio [8], [9], defined as a

Manuscript received May 29, 2009; revised August 04, 2009. First published August 28, 2009; current version published October 09, 2009. This work was supported in part by the Australian Research Council (ARC) and in part by the Research Grants Council of the Hong Kong SAR, China (Project No. CityU 121507). This paper was presented in part at the 14th OptoElectronics and Communications Conference (OECC 2009), Hong Kong, July 2009.

E. W. M. Wong and M. Zukerman are with the Department of Electronic Engineering, City University of Hong Kong, Hong Kong Special Administrative Region (SAR), PRC (e-mail: eeewong @ cityu.edu.hk; m.zu@ cityu.edu.hk).

J. Baliga is with the ARC Special Research Centre for Ultra-Broadband Information Networks (CUBIN), Department of Electrical and Electronic Engineering, The University of Melbourne, Victoria 3010, Australia and also with National ICT Australia, Victoria 3010, Australia (e-mail: jbaliga@ gmail. com).

A. Zalesky is with the University of Melbourne, Victoria 3010, Australia (e-mail: azalesky@unimelb.edu.au).

G. Raskutti is with the Department of Statistics, University of California at Berkeley, Berkeley, USA (e-mail: garvesh@gmail.com).

Color versions of one or more of the figures in this paper are available online at http://ieeexplore.ieee.org.

Digital Object Identifier 10.1109/JLT.2009.2031123 ratio of the bursts/packets that are lost to the bursts/packets that are sent. The main cause of loss is lack of sufficient network resources as losses due to physical layer errors are negligible. Our new method is based on a recently published technique for estimation of blocking probabilities in general overflow loss networks [10]-[12].

The network model that we consider is sufficiently general to be applicable to a number of traffic management techniques shown to reduce blocking in all-optical networks. We include deflection routing [8], [13]-[23] and full wavelength conversion [24]. In addition, we include trunk reservation to account for some of the instability introduced by deflection routing [23], [25]. We focus on a model of a bufferless optical switched network [26]. The study of a bufferless optical switched network is important because although there have been some improvements in optical buffering technologies [1], significant size and energy consumption limitations remain [27]. On the other hand, the alternative of electronic buffering suffers from the drawback of energy intensive optical-to-electrical and electrical-to-optical conversion [27].

Henceforth, we will use the term burst to refer to both a burst in the context of bufferless OBS/JIT networks, and a packet in the context of OPS networks. In Section V.H.1, we demonstrate that the accuracy of our method is not significantly affected by the size of the burst/packet although long bursts introduce dependency between load on consecutive trunks which slightly increases the end-to-end blocking probability. This effect is not captured by our approximation which is therefore slightly more accurate for OPS than for OBS. Accordingly, we will henceforth use the term OBS to refer to both OBS/JIT and OPS.

Our analysis focuses on burst based networks [23], where each node only ensures that there is sufficient capacity on the next link in the route. Bursts that are blocked at nodes other than at their source node consume network resources before they are blocked and cleared from the system. The consumption of resources by blocked bursts has a non-negligible effect on the blocking probability of the network, particularly in networks with a high traffic load. This is unlike circuit-switched or optical circuit-switched (OCS) networks, where the network ensures that there is sufficient capacity on all links in the route and reserves this capacity for the duration of the transmission [5], [12]. In OCS networks, the consumption of network resources by transmissions that are blocked is limited to the circuit set-up time and this wastage of network resources is negligible. This key difference between OCS networks and OBS networks means that analytical models for OCS networks cannot be directly applied to OBS networks and vice versa. 


\section{BLOCKING IN OVERFLOW LOSS NETWORKS}

Calculating blocking probabilities in an overflow loss network model is an age-old tele-traffic problem that still remains pertinent to present-day all-optical networks. For example, an OBS network can be modeled as an overflow loss network if bursts are permitted to overflow to an alternate trunk when all channels comprising the first choice trunk are busy. This is referred to as deflection routing.

The simplest possible overflow loss network comprises a first choice trunk and one or more alternate trunks. We refer to this as the basic model. The basic model serves as a building block for the modeling of large-scale networks that are interconnected via an arbitrary topology. In particular, the basic model can be straightforwardly incorporated into the Erlang fixed-point approximation to facilitate calculation of end-to-end blocking probabilities. Therefore, accurate calculation of end-to-end blocking probabilities in an overflow loss network hinges on accurate calculation of blocking in the basic model.

Blocking analysis of the basic model can be traced back to the work of Kosten [28] in 1937. Kosten derived the distribution of the number of busy channels on the first alternate trunk. Assuming without loss of generality that the mean burst size is equal to unity, bursts were assumed to arrive at the first choice trunk according to a Poisson process with rate $a$. The alternate trunk was assumed to comprise an infinite number of channels. Of particular interest was the mean $M=a E(a, N)$ and the variance $V=M(1-M+a /(N+1-a-M))$ of this distribution, where $N$ is the number of channels composing the first choice trunk and $E(a, N)$ denotes the Erlang-B formula.

More than ten years later, Brockmeyer [29] advanced one step further by deriving the distribution of the number of busy channels on the second alternate trunk, when the first alternate trunk now comprises a finite number of channels, $K$. Brockmeyer showed that the blocking probability on the first alternate trunk is given by the ratio $E(a, N+K) / E(a, N)$.

Generalizing the brute-force derivations of Kosten and Brockmeyer to a cascade comprising more than two alternate trunks is intractable. A seemingly tractable approach soon arose when it was recognized that the arrival of bursts on an alternate trunk was a renewal process. This suggested that existing analysis of the $G I / M / N / N$ queue could be invoked to calculate the blocking probability on the $n$th alternate trunk. Pearce and Potter [30], [31] provided explicit formulae to compute the factorial moments of the distribution of the number of busy channels on an infinite trunk offered the overflow of a $G I / M / N / N$ queue. Although in principle this distribution can be characterized exactly, in practice, numerical methods must resort to matching its first few moments to a specific renewal process (e.g., [32]).

Simpler moment matching techniques arose much earlier. Wilkinson [33] characterized the overflow stream of the $n$th alternate trunk in terms of its variance-to-mean ratio, which is denoted with $Z$ and typically referred to as peakedness. This represents a two-moment match. (Note that $Z=1$ for a Poisson stream.) To estimate the blocking probability perceived by a stream characterized by $(M, Z)$ that is offered to a trunk comprising $N$ channels, Wilkinson suggested to consider this stream to be the overflow stream of a fictitious trunk comprising $x$ channels that is offered a Poisson stream of intensity $a$. Then upon calculating the so-called equivalent random parameters $(a, x)$, as prescribed by Jagerman [34], the blocking probability perceived by the stream characterized by $(M, Z)$ is estimated by $E(a, x+N) / E(a, x)$. This is referred to as the equivalent random method or Wilkinsons method. Fredericks [35] suggested a similar two-moment match based on the notion of peakedness.

Kuczura and Bajaj [36] studied an important generalization of the basic model in which multiple heterogenous streams are offered to a common trunk. A combined stream $(M, Z)$ of mean $M=\sum_{i} m_{i}$, variance $V=\sum_{i} v_{i}$ and peakedness $Z=V / M$ is used to characterize the presence of each overflow stream $\left(m_{i}, v_{i}\right)$. Then either the method of [33] or of [35] can be used to calculate the blocking probability, say $p$, perceived by the combined stream. Hence, the mean of the distribution of the number of busy channels on an infinite trunk offered the overflow of this combined stream is $M p$. Accurately apportioning $M p$ to each marginal stream $\left(m_{i}, v_{i}\right)$ is a formidable problem plaguing all of these approximations. The only choice is to resort to one of many empirical formulas [37].

Unlike the case in the old telephone network of the 1950s and 1960s where overflows of calls had been hierarchical, namely, calls that overflow from a given tier in the hierarchy overflow to a higher tier. In such a case, the moment matching approaches, such as in [33], [35], can lead to accurate approximations [38] because the dependencies are unidirectional, namely, congestion in a lower tier affects loading of a higher tier but not vice versa. However, we consider here a non-hierarchical network [37], where increasing overflow from trunk $j$ may increase the load on trunk $k$ which, in turn, may increase overflow from trunk $k$ to $j$, etc. This effect is called mutual overflow (see page 183 of [37]). It increases the dependencies between the loading in the different service groups which makes it harder to obtain accurate approximations for blocking probability.

A classical approach for evaluation of blocking probability in such networks is the well-known Erlang fixed-point approximation (EFPA). This approximation was conceived by Cooper and Katz [4] in 1964 for the analysis of circuit switched telephony networks. It is based on a one-moment match, whereby each overflow stream is characterized solely in terms of its mean, $m_{i}$ (i.e., the mean number of busy channels if the stream were to be offered to an infinite trunk). All streams offered to a common trunk comprising $N$ channels are pooled together to form a combined stream that offers an intensity of $\sum m_{i}$. Traditionally, the blocking probability perceived by the combined stream as well as each marginal stream $i$ is approximated by $E\left(\sum m_{i}, N\right)$. The overflow of each marginal stream $i$ then goes on to offer an intensity of $m_{i} E\left(\sum_{i} m_{i}, N\right)$ to the next alternate trunk.

In this paper we consider an alternative approximation, in addition to EFPA, for the calculation of end-to-end blocking probabilities in OBS networks. The approximation we consider is our recently introduced Overflow Priority Classification Approximation (OPCA) which was first presented in [10], further explained, analyzed and motivated in [11], and applied to blocking probability estimation for circuit-switched trunk reservation networks in [12]. This approximation represents a radically new approach to the analysis of overflow loss networks, in that traditional moment matching techniques play no role. High 
level description of OPCA will be provided in Section IV.A, before the details of the application to OBS networks with deflections is described.

The contributions of this paper are threefold:

- We apply OPCA to analysis of OBS networks with deflection routing employing wavelength reservation. Note that wavelength reservation is analogous to trunk reservation [39] in circuit switched networks and offers protection against the potential instability resulting from excessive deflection routing. See [23] for details.

- We quantitatively evaluate the performance of OPCA and EFPA with respect to OBS over a range of network topologies including fully meshed networks, ring network and the NSF backbone network, over a range of scenarios involving different mean values and distributions of burst size. We observe that the maximum value of these methods, namely, $\max (\mathrm{EFPA}, \mathrm{OPCA})$, is a robust, conservative and accurate blocking probability estimator in most cases.

- We quantify the extent to which deflection routing can enhance the blocking performance and utilization of OBS networks. In this way, we provide a more accurate and scalable approximation to calculate end-to-end blocking probabilities in an OBS network utilizing deflection routing.

The study of OBS networks with deflection routing (which can be viewed as a non-hierarchical overflow network) has been of great interest to many researchers in recent years (see, e.g., [8], [13]-[19], [21]-[23]). Most of these publications study performance by simulations or provide a single node analysis. Zalesky et al. [22], [23] used EFPA to evaluate blocking probability in such networks. Here we enhance the accuracy and robustness of the blocking probability evaluation using $\max (\mathrm{EFPA}, \mathrm{OPCA})$.

\section{THE MODEL}

In this section we describe the network model. We first outline the network structure that we consider and this is followed by a description of the burst switching algorithms used in the network. Finally, we describe how trunk reservation is implemented in the network model.

\section{A. Network Structure}

We consider a network that comprises a set of nodes $\alpha=$ $\{1, \ldots, N\}$ connected by a set of trunks $\mathcal{J}$. Each trunk $j \in \mathcal{J}$ comprises $f_{j}$ fibers, each of which supports $w_{j}$ wavelengths. Therefore, a trunk carries $C_{j}=f_{j} w_{j}$ wavelength channels called links.

Each unique pair of origin and destination nodes form a directional origin-destination (OD) pair, $m$. The set of all OD pairs in the network is denoted $\beta$. We consider directional OD pairs, so $\{i, j\} \in \beta$ represents an OD pair with $i \in \alpha$ being the origin and $j \in \alpha$ the destination, then $\{j, i\}$ and $\{i, j\}$ are two different elements in $\beta$. The traffic demand $\rho_{m}$ of each OD pair $m=\{i, j\} \in \beta$ is composed of bursts transmitted from $i$ to $j$ that follow a Poisson process with parameter $\rho_{m}$. The burst lengths are exponentially distributed with unit mean. We note the well-known result that the blocking probability of an $\mathrm{M} / \mathrm{M} / \mathrm{k} / \mathrm{k}$ system, known as the Erlang B formula, is dependent only on the mean of the service time and it is insensitive to higher moments of the service time distribution. In other words, the Erlang B formula applies to the more general model known as $\mathrm{M} / \mathrm{G} / \mathrm{k} / \mathrm{k}$ [40]-[43]. This important result has been proven by many authors during the last century [41], [43]-[47]. This indicates that the end-to-end results may also not be too sensitive to the distribution of the burst lengths and will mainly depend on their mean. This will be numerically tested in Section V.H.

Let us consider a directional pair of nodes $(i, j)$, where $i, j \in$ $\alpha$. This pair of nodes is not necessarily an OD pair and both $i$ and $j$ can be intermediate nodes in a route between two origin and destination nodes. Let $U(i, j)$ be the set of routes from node $i$ to node $j$. The $k$ route between $i$ and $j$ is denoted $U_{k}(i, j)$, so $U_{k}(i, j) \in U(i, j)$. In networks where a given pair of nodes has more than one route (i.e., $|U(i, j)|>1$ ), one of the route with the least number of hops is referred to as the primary route denoted $U_{0}(i, j)$. All the other possible routes from $i$ to $j$ are referred to as alternate routes.

\section{B. Burst Forwarding}

At source node $i$, all bursts with destination node $j$ are transmitted on the first trunk of the primary route $U_{0}(i, j)$. At each intermediate node, the burst is forwarded on the next trunk in primary route $U_{0}(i, j)$ until it reaches destination node $j$. If at any node, including the source node, all the links on the trunk of the route are unavailable, the burst is deflected onto an alternate route. If the burst is deflected at node $l$, then the set of alternate routes is $U(l, j)-U_{0}(l, j)$. Preference is given to shorter routes followed by pre-assigned ordering. A given burst is permitted to be deflected at most $D$ times. A burst is considered blocked (discarded/lost) if it arrives at a given node where all output trunks are busy or while trying alternate trunks, the burst reaches the maximum allowable number of deflections.

In our model we assume an ideal case with no guard bands between bursts. In addition, we do not consider specific OBS reservation protocols [48], scheduling algorithms [49], or partial wavelength conversion [50]. Finally, we note that the results presented in this paper are equally applicable to a network with no wavelength conversion which has $f_{j} w_{j}$, instead of $f_{j}$, fibers per trunk [51].

\section{Wavelength Reservation}

In a network with wavelength channel reservation, some of the capacity on each trunk is reserved for bursts that have not been deflected [23]. Bursts belonging to OD pair $m$, on the primary route between OD pair $m$, are undeflected bursts. In our network model, we set wavelength channel reservation threshold $T_{j} \leq C_{j}$ on each trunk $j$. If the number of links occupied on trunk $j$ is greater than or equal to $T_{j}$, only undeflected bursts are permitted to use that trunk.

\section{Blocking Probability Estimation Technigues}

In this section we present the techniques we use to estimate blocking probability. The first two are adaptations of EFPA and OPCA to the case of OBS networks with deflections. Then we provide a qualitative discussion that provides insight into the accuracy of these two approaches under various traffic conditions. Finally, we introduce a method based on the maximum of the blocking probability evaluations of EFPA and OPCA and argue 
that it can provide an accurate and almost always conservative blocking probability evaluation.

\section{A. EFPA and OPCA}

Here we describe the algorithms that adapt EFPA and OPCA to the evaluation of blocking probability of OBS networks with deflections. We also outline the specific choices we make in the implementation of the algorithms to obtain the numerical results presented in Section V.

Calculation of blocking probability for both OPCA and EFPA begins by randomly choosing the initial blocking probabilities (Uniform $(0,0.1])$. We then solve a set of fixed-point equations by successive substitutions until convergence occurs. The result of the fixed-point equations is the blocking probability on each link, which is used to calculate the end-to-end blocking probability for each OD pair. The average blocking probability, over all OD pairs, is the weighted average of the end-to-end blocking probability for each OD pair.

The fixed-point equations for EFPA and OPCA can be broken up into two groups. The first group of equations are identical for EFPA and OPCA, and are used to calculate the offered load on each link, using the current estimate of the blocking probability. The second group of equations are used to calculate a new estimate of the blocking probability, using the estimate of offered load calculated using the first group of equations. The second group of equations are different for each estimation method (EFPA and OPCA).

In the following we outline the equations we use to estimate the offered load (common to EFPA and OPCA) and also the equations we use to estimate the blocking probability (different for EFPA and OPCA).

1) Offered Loads: As before, the traffic demand, or offered load, of each OD pair $m$ is $\rho_{m}$. Let $a_{j}^{k}(m)$ be the offered load of OD pair $m \in \beta$, with $k \in\{1, \ldots, D\}$ deflections, on trunk $j \in \mathcal{J}$. Let $b_{j}^{k}$ be the probability that a burst with $k$ deflections is blocked on trunk $j$. If the first trunk of the primary route between OD pair $m$ is trunk $i_{1}$, then trunk $i_{1}$ is offered the full load of the OD pair, i.e., $a_{i_{1}}^{0}(m)=\rho_{m}$. The second trunk $i_{2}$ in the primary route is offered the carried load of the first trunk. The carried load is defined as the proportion of offered load that is not blocked. Therefore, the offered load of OD pair $m$ on the second trunk $i_{2}$ of the primary route is

$$
a_{i_{2}}^{0}(m)=\rho_{m}\left(1-b_{i_{1}}^{0}\right)
$$

On the other hand, due to congestion, bursts are occasionally blocked on a trunk of the primary route and are deflected onto alternate trunks and routes. The load offered to the first trunk $l_{1}$ of the first choice alternative route is related to the load offered to trunk $i$ on the primary route by

$$
a_{l_{1}}^{k+1}(m)=a_{i}^{k}(m) b_{i}^{k}
$$

where $k$ is the number of deflections prior to the latest deflection. Similarly, the load offered to the first trunk $l_{2}$ of the second choice alternative route is

$$
a_{l_{2}}^{k+2}(m)=a_{l_{1}}^{k+1}(m) b_{l_{1}}^{k+1}=a_{i}^{k}(m) b_{i}^{k} b_{l_{1}}^{k+1} .
$$

2) Blocking Probabilities: Let $a_{j}^{k}$ be the offered load, with $k$ deflections, on trunk $j$. The variables $a_{j}^{k}$ and $a_{j}^{k}(m)$ are related by

$$
a_{j}^{k}=\sum_{m \in \beta} a_{j}^{k}(m) .
$$

In addition, let $\tilde{a}_{j}^{k}$ be the offered load, with up to and including $k$ deflections, on trunk $j$. The variables $\tilde{a}_{j}^{k}$ and $a_{j}^{k}$ are related by

$$
\tilde{a}_{j}^{k}=\sum_{l=0}^{k} a_{j}^{l}(m)
$$

EFPA: We evaluate the link state probability $q_{j}(i)$ for each trunk $j \in \mathcal{J}$ and each state $i \in\left\{1, \ldots, C_{j}\right\}$ (i.e., $i$ links occupied) using

$$
q_{j}(i)=\left(a_{j}^{0}+1\left\{T_{j}>i-1\right\} \sum_{n=1}^{D} a_{j}^{n}\right) q_{j}(i-1) / i
$$

where 1\{\} is the indicator function and $q_{j}(0)$ is set such that $\sum_{i=0}^{C_{j}} q_{j}(i)=1$ is satisfied.

The blocking probability, for bursts with $k \in\{0, \ldots, D\}$ deflections, on trunk $j$ is estimated by

$$
b_{j}^{k}=\left\{\begin{array}{ll}
q_{j}\left(C_{j}\right), & k=0 \\
\sum_{i=T_{j}}^{C_{j}} q_{j}(i), & k \geq 1
\end{array} .\right.
$$

Equations (1)-(7) form a set of fixed-point equations which can be solved by successive substitutions using an algorithm similar to the one used in [23].

OPCA: OPCA is fundamentally different from EFPA. It is able to capture dependency between traffic on different trunks, but it still decouples between trunks to achieve a scalable approximation. It works by using a surrogate second system and estimating the blocking probability in the second system by EFPA. Although the surrogate system may be different from the real system we aim to analyze, the application of EFPA to the surrogate system will provide a better blocking probability approximation for the original problem than application of EFPA to the original problem. The surrogate system is defined by regarding an overflow loss network as if it were operating under a preemptive priority regime where each call is classified according to the number of times it has overflowed and junior bursts (bursts that experienced less overflows) are given priority. By giving priority to junior bursts, the senior bursts that have more "memory" about busy trunks, namely, trunks where all links are busy, are preempted and overflowed and these senior bursts will only attempt trunks which they did not visit before. This way, during a period of congestion, these senior bursts are likely to run out of trunks to visit and they will be blocked and cleared from the system when they reach they maximum allowable number of deflections. During light traffic periods, following after an occasional preemption, the deflected senior burst is likely to have many choices of trunks to attempt and is not likely to be blocked. In this way, OPCA captures trunk load dependencies as in the real system. This is fundamentally different from EFPA that ignores trunk dependencies and treat 
each trunk as loaded with the same Poisson traffic regardless of loading of other trunks.

Because under OPCA, "junior" bursts are given priority, the priority of a burst is incrementally reduced on each occasion it overflows. We remind the reader that this prioritization is artificially introduced in the surrogate system to obtain a more accurate approximation and it is not a feature of the real network. A comprehensive set of rigorous and intuitive arguments as well as numerical results were presented in [11], [12] to explain and demonstrate the benefit of OPCA over EFPA.

We evaluate the link state probability $p_{j}^{k}(i)$ for each trunk $j \in \mathcal{J}$, for $k \in\{0, \ldots, D\}$ deflections and each state $i \in$ $\left\{1, \ldots, C_{j}\right\}$ using

$$
p_{j}^{k}(i)=\left(a_{j}^{0}+1\left\{T_{j}>i-1\right\} \sum_{n=1}^{k} a_{j}^{n}\right) p_{j}^{k}(i-1) / i
$$

where $p_{j}^{k}(0)$ is set such that $\sum_{i=0}^{C_{j}} p_{j}^{k}(i)=1$ is satisfied.

The average blocking probability $\bar{b}_{j}^{k}$ on trunk $j \in \mathcal{J}$, for bursts with up to and including $k \in\{1, \ldots, D\}$ deflections, is estimated by

$$
\bar{b}_{j}^{k}=\frac{\sum_{n=0}^{k}\left(a_{j}^{n} \sum_{i=T_{j}}^{C_{j}} p_{j}^{k}(i)\right)}{\tilde{a}_{j}^{k}}
$$

and $\bar{b}_{j}^{0}$ is estimated using the Erlang-B formula, i.e., $\bar{b}_{j}^{0}=E\left(a_{j}^{0}, C_{j}\right)$. The blocking probability, for bursts with $k \in\{0, \ldots, D\}$ deflections, on trunk $j$ is estimated by

$$
b_{j}^{k}= \begin{cases}\bar{b}_{j}^{0}, & k=0 \\ \frac{\bar{b}_{j}^{k} \tilde{a}_{j}^{k}-\bar{b}_{j}^{k-1} \tilde{a}_{j}^{k-1}}{a_{j}^{k}}, & 1 \leq k \leq D .\end{cases}
$$

Note that the blocking probability of undeflected bursts is calculated using the Erlang-B formula.

To obtain the OPCA blocking probability estimates, we start with the primary traffic, i.e., $k=0$. Then, we solve the fixedpoint equations described by (1), (4) and (8), by successive substitutions, to obtain the $p_{j}^{0}(i)$ values for $j \in \mathcal{J}$ and $i \in$ $\left\{1, \ldots, C_{j}\right\}$, as well as the values $a_{j}^{0}$ for $j \in \mathcal{J}$ and $\bar{b}_{j}^{0}=b_{j}^{0}=$ $E\left(a_{j}^{0}, C_{j}\right)$.

Next, having all the parameters related to the primary traffic $(k=0)$, we progress to compute the parameters associated with the first deflection traffic $(k=1)$. We solve the fixed-point equations (2), (3), (4), and (8) to obtain the $p_{j}^{1}(i)$ values for $j \in \mathcal{J}$ and $i \in\left\{1, \ldots, C_{j}\right\}$, as well as the values $a_{j}^{1}$ for $j \in \mathcal{J}$, and $\bar{b}_{j}^{1}$ and $b_{j}^{1}$ using (9) and (10), respectively, for every $j \in \mathcal{J}$, where $\tilde{a}_{j}^{1}$ is given by (5).

Then, having all the parameters related to the primary and the first deflection traffic ( $k=0$ and $k=1$ ), we compute the parameters associated with the second deflection traffic $(k=2)$.

The process of deriving the parameters for $k=2$ repeats itself until we have all the parameter values for all $k \in\{1, \ldots, D\}$.

3) End-to-End Blocking Probabilities: Let $\mathcal{E}_{m} \subset \mathcal{J}$ be the set of trunks connected to the destination node of OD pair $m$. The end-to-end blocking probability of OD pair $m, P_{m}$, is estimated by

$$
P_{m}=1-\frac{\sum_{j \in \mathcal{E}_{m}} \sum_{k=0}^{D} a_{j}^{k}(m)\left(1-b_{j}^{k}\right)}{\rho_{m}}
$$

where $a_{j}^{k}(m)\left(1-b_{j}^{k}\right)$ is the carried load of OD pair $m$, with $k$ deflections, on trunk $j$.

The average blocking probability for the network $P$ is estimated by

$$
P=\frac{\sum_{m \in \beta} \rho_{m} P_{m}}{\sum_{m \in \beta} \rho_{m}} .
$$

\section{B. EFPA Versus OPCA for Different Traffic Regions}

In low traffic region, OPCA is accurate and EFPA underestimates the blocking probability. As discussed in [11], OPCA captures well the dependency and occurrences of occasional congestion, while EFPA suffers from errors due to the Poisson and independence assumptions.

By comparison, during a very heavy traffic period, overflow traffic dominates and links are used inefficiently because bursts take longer paths using more links. OPCA considers a surrogate system that gives preemption priority to the primary traffic, so during such heavy traffic load, the primary traffic that use short paths dominates, when in fact the overflow traffic dominates in the real system. Therefore OPCA underestimates the blocking in this very heavy traffic region. On the other hand, EFPA (like the real system) does not give priority to primary traffic, so it can capture the effect of the dominating overflow traffic (longer paths and lower carried load) and therefore it predicts higher blocking probability than OPCA.

Then there is a third intermediate period where the traffic load is in a region between the above two extreme regions. This represents the case where we have sufficiently heavy traffic and in the real system there is a potentially significant drop in the carried traffic while in the EFPA, the fixed-point solution is not unique (see Figs. 3 and 5 in [23]). OPCA solution is unique in this intermediate region because it, as discussed, discriminates against overflowed traffic. Henceforth, we call this intermediate region an unstable region. The reader is referred to [23] for further discussion on this intermediate unstable region.

\section{C. $\max (E F P A, O P C A)$}

The different behaviors of EFPA and OPCA give rise to a new approximation based on choosing the maximal value of the EFPA and OPCA blocking probability approximations designated $\max (\mathrm{EFPA}, \mathrm{OPCA})$. As demonstrated empirically in Section V, $\max$ (EFPA, OPCA) can lead to accurate approximations in most scenarios, and almost always it seems to be a conservative approximation.

\section{NuMERICAL RESULtS}

The performance of OPCA is evaluated and compared to the performance of EFPA using simulations. We consider ring networks, fully meshed networks and the 13-node National Science Foundation network (NSFNET). The NSFNET is often chosen to represent a core national network, and the ring and fully meshed topologies are two extreme network topologies that are often used as physical or logical topologies. To limit excessive simulation times we focus on traffic loads that result in blocking probabilities above $10^{-5}$. In our simulations the burst arrival process for each OD pair follows a Poisson process as in our analytical modelling. Also, as in our analysis routing preference is 


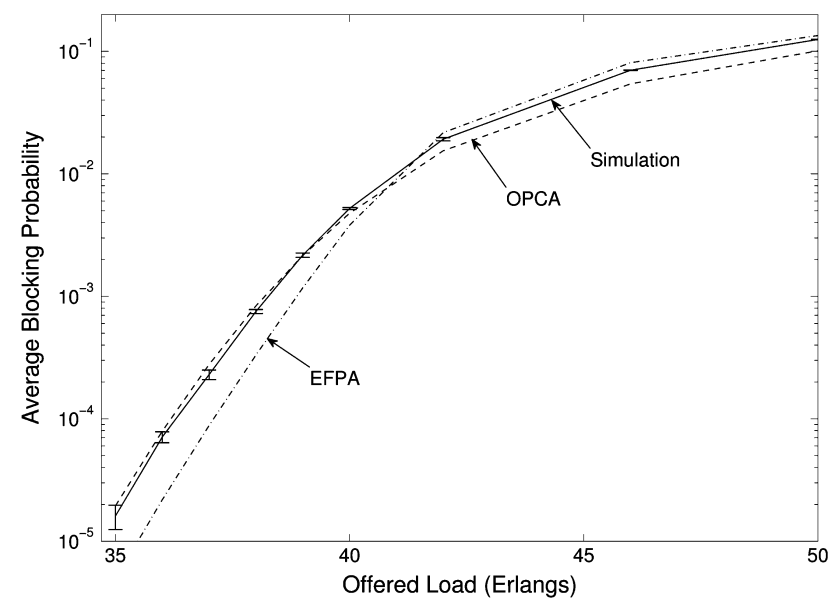

Fig. 1. Blocking probability of OPCA and EFPA vs. simulation results for a 6-node fully meshed topology with $90 \%$ reservation threshold, and the number of deflections is limited to 4 .

given to shorter routes followed by pre-assigned ordering. The pre-assigned order is chosen at random before the simulation runs and it remains unchanged. Then the corresponding analytical results are based on the same order.

The difference between the simulation and the analytical modelling is that the simulation does not make the trunk independence assumption. As such, the simulation also does not assume the process of overflowing bursts at each trunk to be Poisson. We do not simulate the intricacies of the burst reservation process (e.g., bursts do not seek to reserve wavelength resources before their arrival, as per the just-enough-time scheme [52]). Instead, we assume a burst seeks a wavelength at the instant it arrives as in the JIT scheme [6]. In this way, the simulation exclusively tests the error introduced by the independence and the Poisson assumptions.

Our simulation also allows for a given burst, assuming it is long enough, to be transmitted simultaneously on multiple consecutive trunks (analyzed in [53] only for the special case of a network composed of optical cross-connects connected in tandem). This phenomenon is not captured by our analytical modelling which assumes decoupling and independence of the various trunks.

\section{A. Fully Meshed Network Topologies}

Fig. 1 shows the average blocking probability $(P)$ in a 6-node fully meshed network for average offered loads, $\rho_{m}$ from 35 to 50 erlangs. Each trunk has 50 links and each node in the network forms an OD pair with every other node in the network, giving a total of 30 OD pairs. The wavelength channel reservation threshold is set to 45 for each trunk. The results indicate that OPCA is accurate and conservative for offered loads from 35 to 40. OPCA underestimates the blocking probability when the offered load is greater than 40 . On the other hand, EFPA gives accurate and conservative results for offered loads greater than 42 , but underestimates the blocking probability when the offered load is less than 42 . We observe that for this case, $\max (\mathrm{EFPA}, \mathrm{OPCA})$ provides the best approximation which is also conservative except for loads between 40 and 42 erlangs.

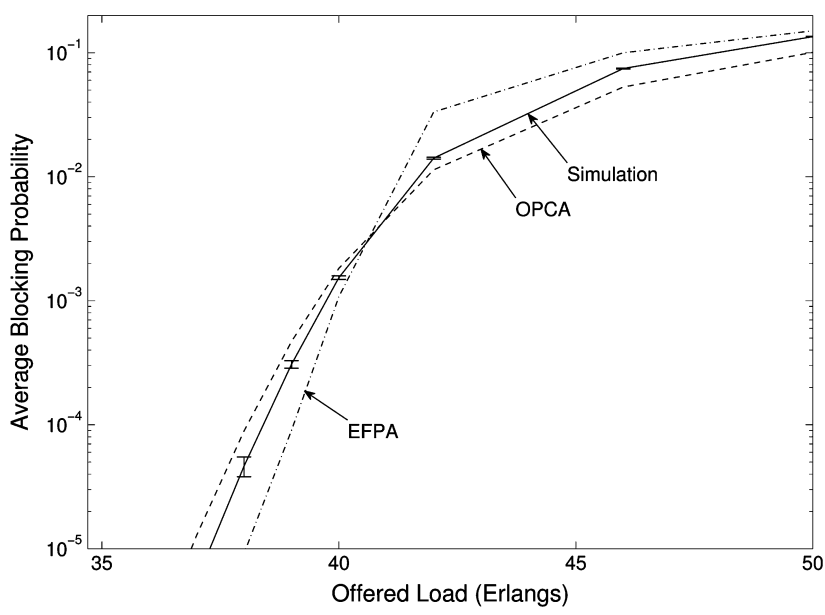

Fig. 2. Blocking probability of OPCA and EFPA vs. simulation results for a 8 -node fully meshed topology with $90 \%$ reservation threshold, and the number of deflections is limited to 6 .

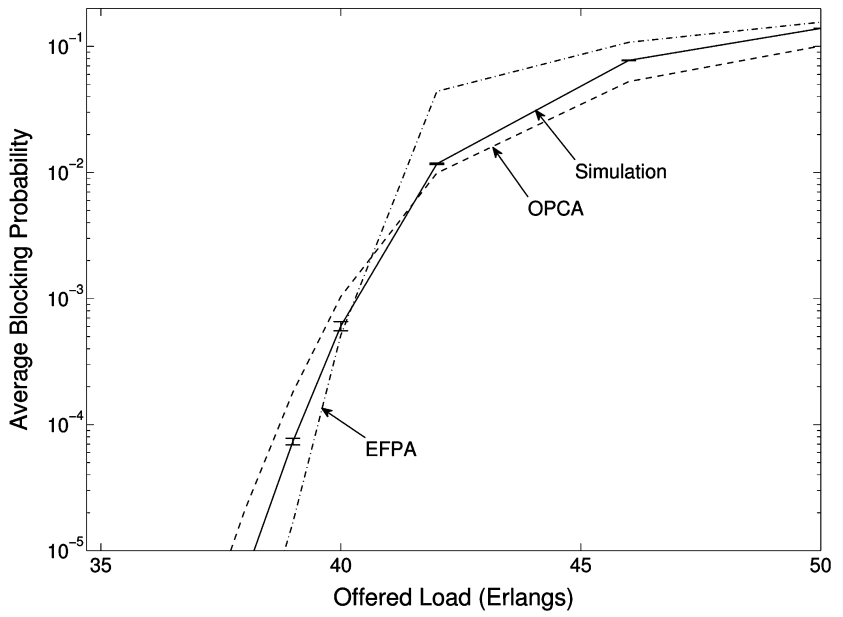

Fig. 3. Blocking probability of OPCA and EFPA vs. simulation results for a 10 -node fully meshed topology with $90 \%$ reservation threshold, and the number of deflection is limited to 8 .

In Figs. 2 and 3 we consider 8- and 10-node fully meshed networks, respectively, and again compare between OPCA and EFPA. For the 8-node the maximal number of deflections increase to 6 , while in the 10-node network it increases to 10 . We observe that as the number of nodes and the maximal number of deflections increases, the EFPA approximation tends to overestimate the blocking probability for high loads, and then as in the case of the 6-node network, underestimate in the case of low loads.

Then in Fig. 4, we consider the 10-node fully meshed topology where the reservation threshold is extended to $80 \%$. Lowering the reservation threshold, provides greater protection for new (undeflected) flows. This suppresses the unstable region, and reduces overflow traffic, which in turn decreases the Poisson and independence errors (which improves accuracy of both EFPA and OPCA). In addition, OPCA gives priority to un-deflected bursts, which is consistent with the effect of an aggressive reservation threshold. This results in higher accuracy of OPCA. As a result, $\max (\mathrm{EFPA}, \mathrm{OPCA})$ is more accurate than in the case of a $90 \%$ reservation threshold. 


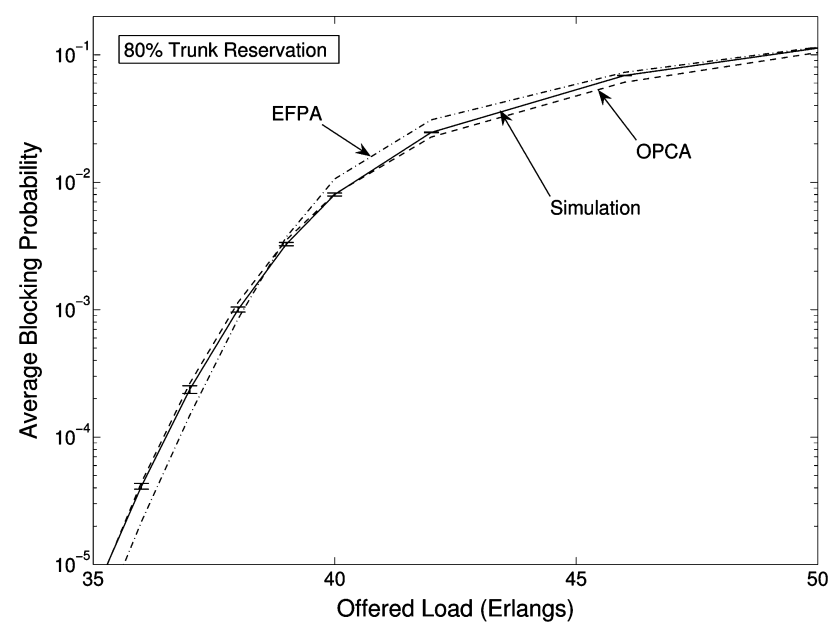

Fig. 4. Blocking probability of OPCA and EFPA vs. simulation results for a 10-node fully meshed topology with $80 \%$ reservation threshold, and the number of deflection is limited to 8 .

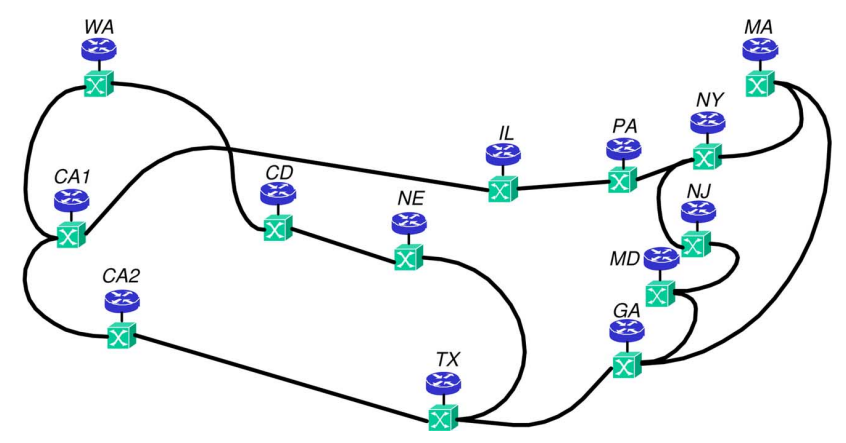

Fig. 5. NSF network topology, each solid line represents two uni-directional trucks in opposing directions.

Notice that in the scenario presented in Fig. 3, the $\max ($ EFPA, OPCA) loses certain accuracy in the high load range where EFPA over-estimates the blocking probability, but again, it is almost always conservative. As we use blocking probability estimations for dimensioning purposes, the fact that $\max (\mathrm{EFPA}, \mathrm{OPCA})$ may overestimate the blocking probability for certain very high loads (low performance-and probably unstable) range is not a significant issue because this range must be avoided. Notice that for acceptable blocking probabilities such as $10^{-3}$ or $10^{-4}$, the error introduced by $\max (\mathrm{EFPA}, \mathrm{OPCA})$ is small in terms of error in dimensioning. Even for the most inaccurate scenario of the 10 node network, if we aim to dimension a network based on say $10^{-3}$ blocking probability and use the $\max (\mathrm{EFPA}, \mathrm{OPCA})$ approximation for this purpose, we will only over-dimension the network by some $2 \%$ which is an acceptable error especially given the much larger errors in traffic prediction.

\section{B. NSF Network}

We consider an NSF network with 13 nodes and 32 trunks. The topology of the NSF network is shown in Fig. 5. As before, each trunk has 50 links and the wavelength channel reservation threshold is set to 45 for each trunk. We randomly select a set of $12 \mathrm{OD}$ pairs and simulate the network for average offered loads, $\rho_{m}$ from 15 to 50 erlangs. Figs. 6 and 7 show the average

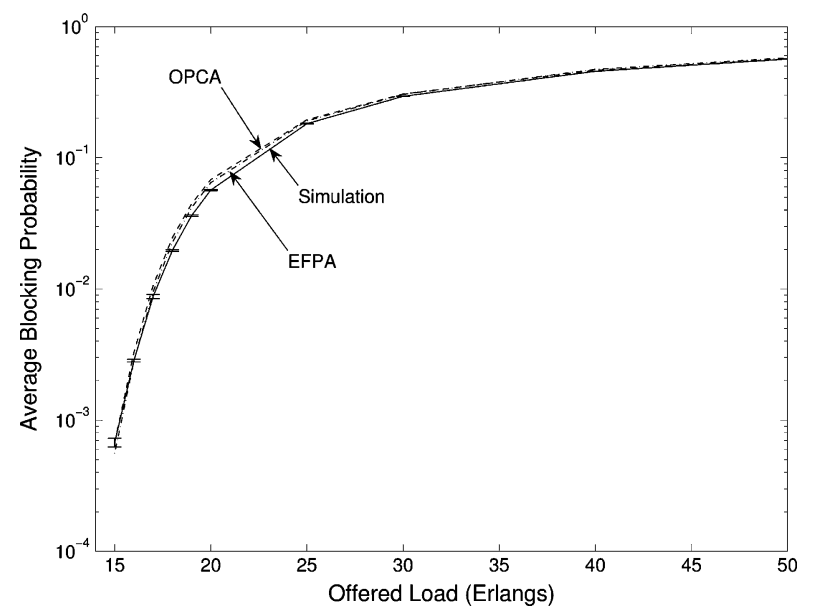

Fig. 6. Blocking probability comparison of OPCA vs. EFPA for NSF topology with $90 \%$ reservation threshold. The OD pairs are according to Set 1 in Table II.

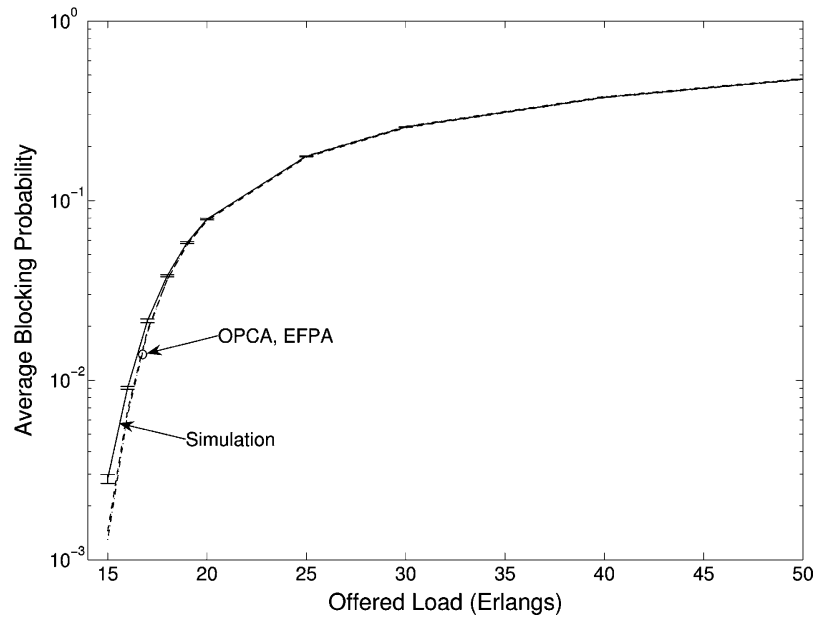

Fig. 7. Blocking probability comparison of OPCA vs. EFPA for NSF topology with $90 \%$ reservation threshold. The OD pairs are according to Set 2 in Table II.

blocking probability of the network, obtained using simulations, when the sets of OD pairs are according to Set I and Set II, respectively from Table II. We also present in these two figures the corresponding blocking probability estimates using OPCA and EFPA. The blocking probability estimated using OPCA is very close to that obtained using EFPA. In addition, the simulation results indicate that the blocking probability estimates obtained using either OPCA or EFPA are accurate. Thus max(EFPA, OPCA) is also accurate.

These results demonstrate that $\max (\mathrm{EFPA}, \mathrm{OPCA})$ does not improve significantly the already accurate results of EFPA in networks that are not heavily meshed. Nevertheless, it is important to have available a method which is robust and is not dependent on topology. Based on all the results we present, $\max (\mathrm{EFPA}, \mathrm{OPCA})$ satisfies this requirement.

\section{6-Node Ring Network}

Fig. 8 shows the average blocking probability in a 6-node bi-directional ring network for average offered loads, $\rho_{m}$, varied between 7 and 10 erlangs. Each trunk has 50 links and each node in the network forms an OD pair with every other node in the network, giving a total of 30 OD pairs. As with the 6-node fully 


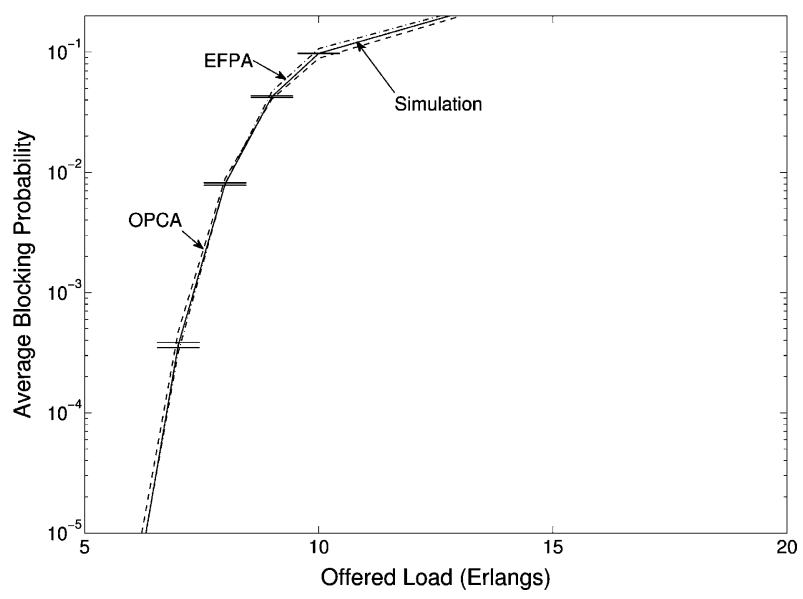

Fig. 8. Blocking probability comparison of OPCA vs. EFPA for a 6-node ring topology with $90 \%$ reservation threshold.

TABLE I

\section{LIST OF NOTATION}

\begin{tabular}{ll}
\hline \hline$a_{j}^{k}(m)$ & offered load of OD pair $m$, with $k$ deflections, on trunk $j$ \\
$a_{j}^{k}$ & offered load, with $k$ deflections, on trunk $j$ \\
$\tilde{a}_{j}^{k}$ & offered load, up to $k$ deflections, on trunk $j$ \\
$b_{j}^{k}$ & blocking probability of link $j$ for traffic with $k$ deflections \\
$q_{j}(i)$ & probability of state $i$ for link $j$ (EFPA) \\
$p_{j}^{k}(i)$ & probability of state $i$ for link $j$, with $k$ deflections (OPCA) \\
\hline \hline
\end{tabular}

TABLE II

Two SETS OF INGRESS AND EGRESS ROUTER PAIRS

\begin{tabular}{||c|c||c|c||}
\hline \hline \multicolumn{2}{|c||}{ Set 1 } & \multicolumn{2}{c||}{ Set 2 } \\
\hline Ingress & Egress & Ingress & Egress \\
\hline WA & MD & NJ & NE \\
CA1 & IL & IL & NY \\
CA1 & MA & CA2 & WA \\
CA2 & MA & WA & PA \\
TX & NY & CA2 & TX \\
GA & MA & CA2 & PA \\
MD & WA & CA1 & GA \\
IL & CA1 & MD & NJ \\
MA & CA1 & MA & PA \\
MA & CA2 & CA2 & CA1 \\
NY & TX & NE & GA \\
MA & GA & PA & CD \\
\hline \hline
\end{tabular}

meshed and NSF networks, the wavelength channel reservation threshold is set to 45 for each trunk. The blocking probability estimated using OPCA is very close to that obtained using EFPA. In addition, as is the case for the NSF Network, the simulation results indicate that the blocking probability estimates obtained using either OPCA or EFPA are accurate.

\section{Blocking Versus Number of Deflections and Trunk Size}

We present here results for the blocking probability over a range of values for the maximal allowable number of deflections and the trunk size (namely, the number of links per trunk). The aims are to study the effects of the allowable number of deflections and trunk size on blocking probability and to evaluate the accuracy of our max(EFPA, OPCA) approximation. To evaluate the accuracy of $\max (\mathrm{EFPA}, \mathrm{OPCA})$ against the

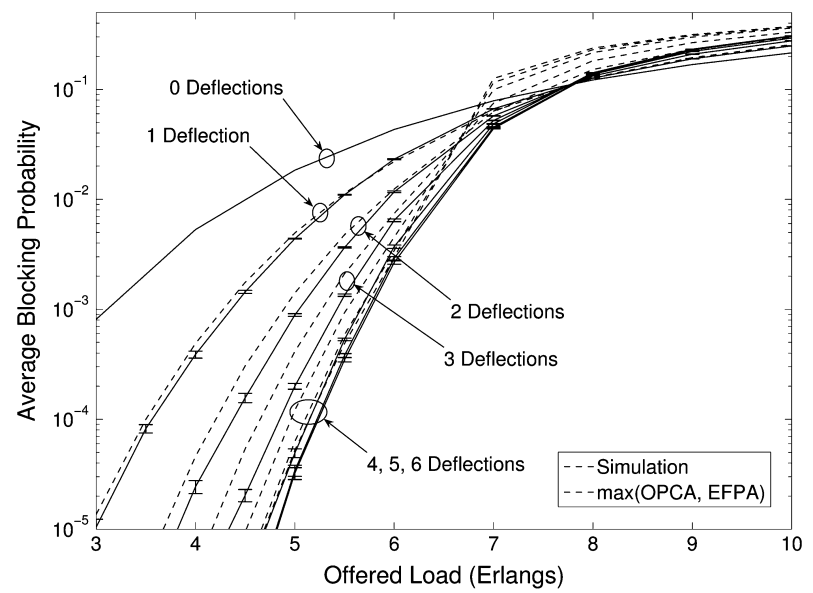

Fig. 9. Blocking probability evaluations based on simulations and $\max (\mathrm{EFPA}, \mathrm{OPCA}$ ) estimates for a 6-node fully meshed network with maximal allowable number of deflections ranges between $0-6$. Each trunk in the network has 10 links with a reservation threshold of $90 \%$.

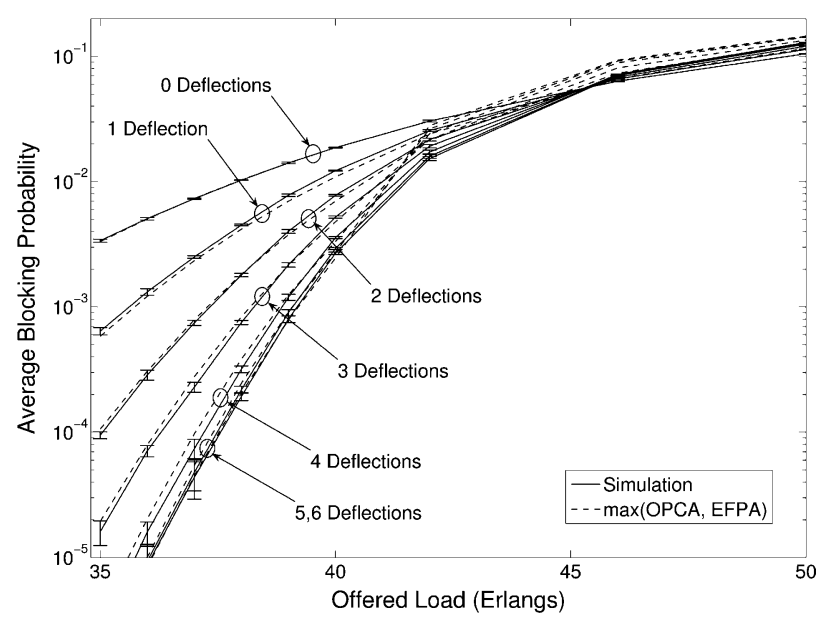

Fig. 10. Blocking probability evaluations based on simulations and $\max ($ EFPA, OPCA) estimates for a 6-node fully meshed network with maximal deflections between 0-6. Each trunk in the network has 50 links with a reservation threshold of $90 \%$.

number of deflections allowed and trunk size, we focus on a fully meshed network because the low average nodal degree in ring networks and the NSF network restricts the alternate paths and therefore the number of deflections [25]. We consider a reservation threshold of $90 \%$ and vary the maximal allowable number of deflections between $0-6$. For trunk size values, we consider 10, 50 and 100 links per trunk.

In Figs. 9, 10, and 11, we present results for the blocking probability evaluations based on simulations and $\max (\mathrm{EFPA}, \mathrm{OPCA})$ estimates for the cases where the numbers of links per each trunk in the network has 10,50 and 100 links respectively. The $95 \%$ confidence intervals based on Student's t-distribution are also provided for the simulation results. We can see that the $\max (\mathrm{EFPA}, \mathrm{OPCA})$ estimates the simulation results reasonably well, although in many cases the estimates fall outside the confidence intervals. We also observe reduction in marginal benefit as the number of allowable deflections increases. There is a clear benefit in our example to increase the maximal number of deflection all the way to 


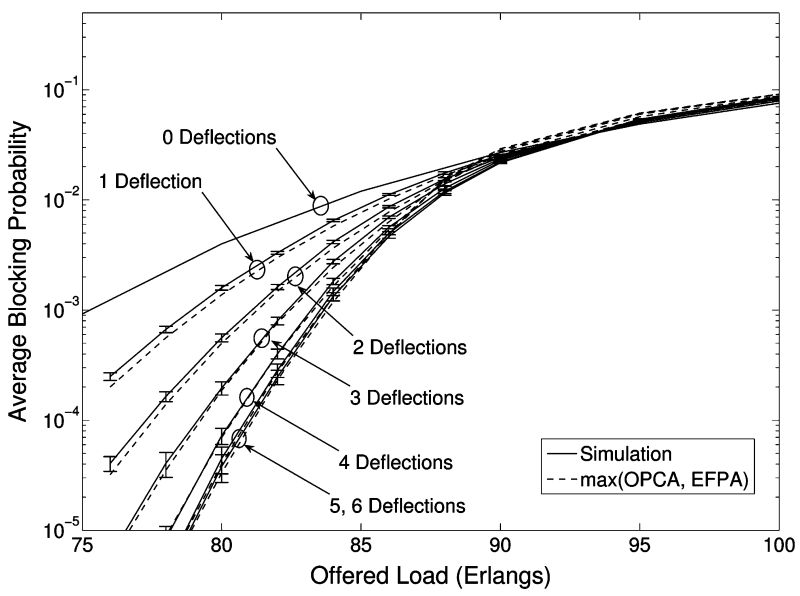

Fig. 11. Blocking probability evaluations based on simulations and $\max (\mathrm{EFPA}, \mathrm{OPCA})$ estimates for a 6-node fully meshed network with maximal deflections between $0-6$. Each trunk in the network has 100 links with a reservation threshold of $90 \%$.

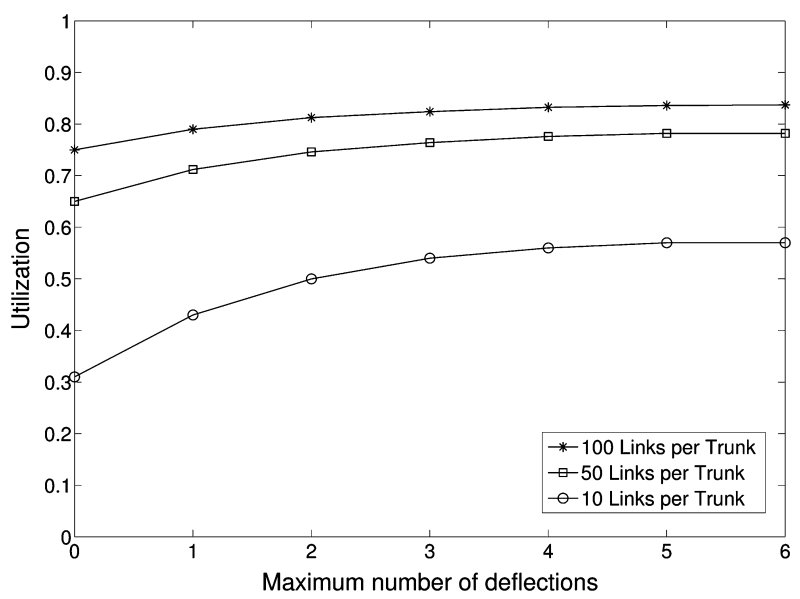

Fig. 12. The utilization of a 6-node fully meshed network with reservation threshold of $90 \%$, target blocking probability of $10^{-3}$ and maximal deflections between $0-6$ when the number of links per trunk is 10,50 and 100 .

four. However, increasing this number to five or six, does not significantly reduce the blocking probability.

\section{E. Utilization}

An important measure to a designer is resource utilization. We target blocking probability of $10^{-3}$ and aim to find the maximal achievable utilization such that the blocking probability will not exceed $10^{-3}$. In Fig. 12, we provide a plot of the simulated average blocking probability (using the results of the previous figures) against offered load for 0-6 maximal deflections. The results show that increasing the number of deflections can provide significant utilization gain in fully meshed OBS networks. This is especially true for the cases where the number of links per trunk is low, where only low utilization is achievable in the case of no deflections. We also observe again the reduction in marginal benefit. Clearly, beyond four deflections the additional utilization gain for the present case is minor.

Next, we investigate if the above results on utilization achieved for a 6-node network are applicable if the size of the network increases. In Fig. 13 we present utilization results (again, based on simulated average blocking probability using

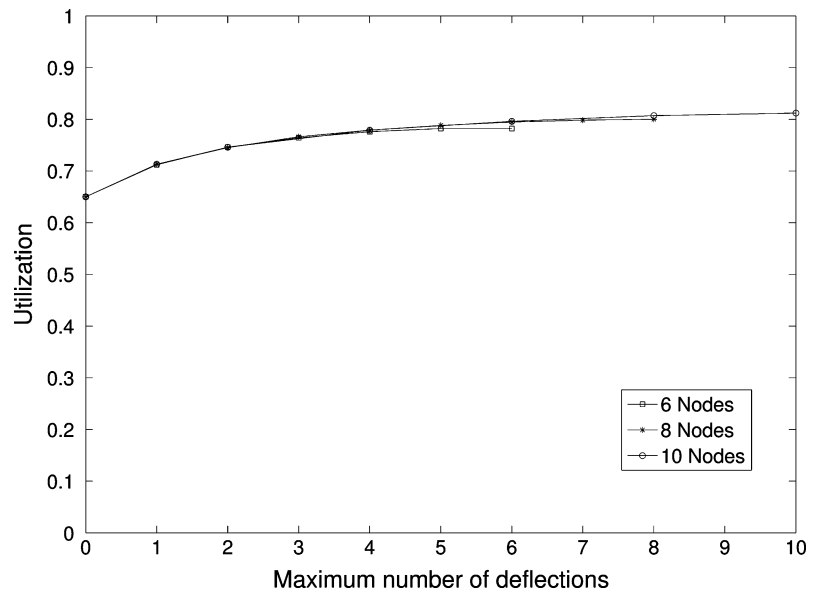

Fig. 13. The utilization of a 6,8 and 10-node fully meshed networks with 50 links per trunk and reservation threshold of $90 \%$, target blocking probability of $10^{-3}$ and maximal deflections between $0-6,0-8$ and $0-10$ for the 6,8 and 10-node networks, respectively.

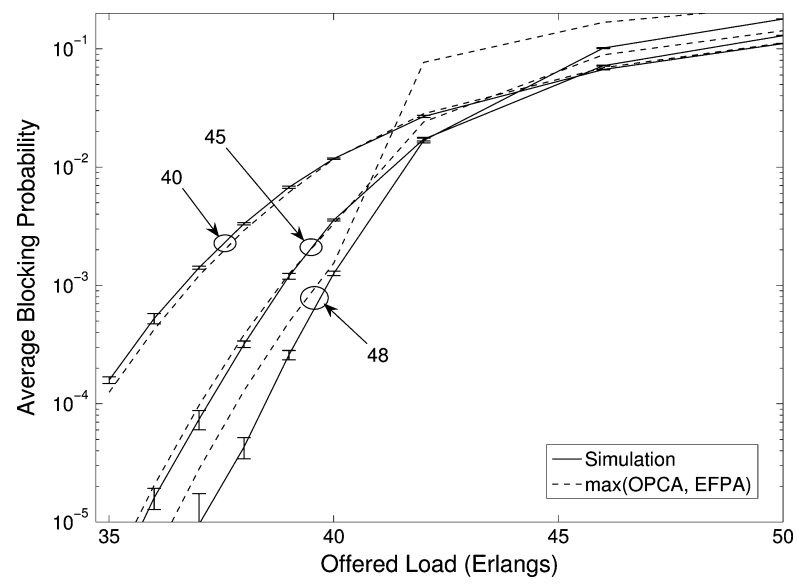

Fig. 14. Blocking probability comparison of $\max (\mathrm{OPCA}, \mathrm{EFPA})$ versus simulation results for a 6-node fully meshed topology with $80 \%$ (denoted 40), $90 \%$ (denoted 45 ) and $96 \%$ (denoted 48) reservation thresholds.

the results of the previous figures) for a 6-node fully-meshed network, an 8-node fully-meshed network and a 10-node fullymeshed network, each with 50 links per trunk and 90\% reservation thresholds. As before, we target a blocking probability of $10^{-3}$. We observe that in the example presented, the network size does not significantly affect the utilization results.

\section{F. Threshold}

We have already demonstrated that having lower threshold (more protection for undeflected bursts) leads to better accuracy of $\max (\mathrm{EFPA}, \mathrm{OPCA})$. We now further examine the effect of threshold level on the accuracy of $\max (\mathrm{EFPA}, \mathrm{OPCA})$.

In Fig. 14, we consider a fully meshed network with six nodes and 50 links per trunk and vary the traffic load and the threshold level. We observe that for the case of $80 \%$ reservation threshold, represented by the curves denoted 40 , the accuracy of $\max (\mathrm{EFPA}, \mathrm{OPCA})$ is excellent. Fairly good accuracy is achieved in the case denoted 45 where we have $90 \%$ reservation threshold. However, in the case denoted 48 where we have $96 \%$ reservation threshold (almost no reservation), the accuracy of the estimate falls and this is because the network itself becomes unstable due to deflections. 


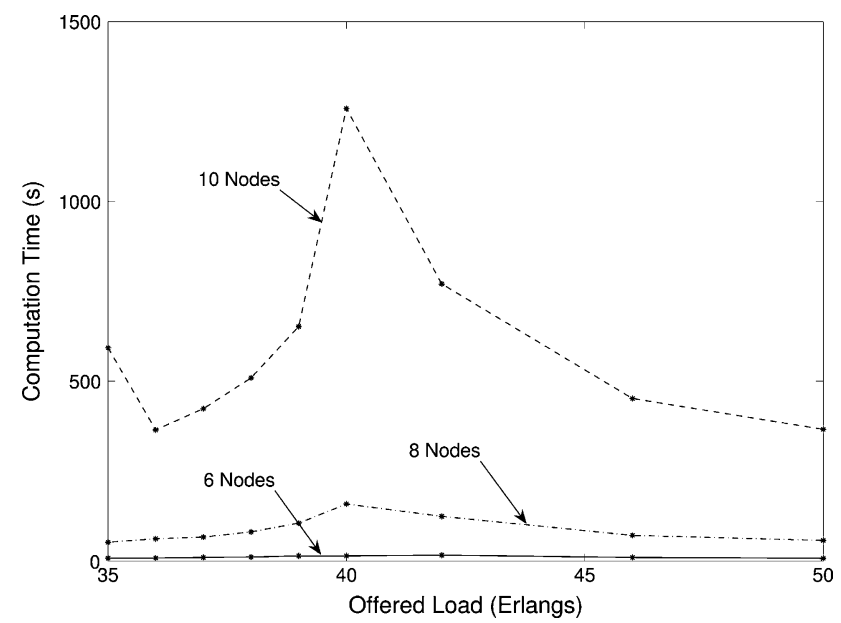

Fig. 15. Running times of EFPA for a 6-node, 8-node and 10-node fully meshed topology with $90 \%$ reservation threshold. The maximal number of deflections is 4,6 and 8 for the 6,8 , and 10 node networks, respectively.

\section{G. Running Time}

Both EFPA and OPCA are based on fixed-point solutions. Because it is difficult to evaluate analytically their convergence rate, we rely on empirical measurements to evaluate and compare their running time. In the examples studied, OPCA has been faster than EFPA as demonstrated in Figs. 15, 16 and 17. Figs. 15 and 16 represent absolute running times while Fig. 17 illustrates the ratios of running times OPCA/EFPA.

From the empirical evidence, the running time of OPCA is clearly no longer than that of EFPA. This implies that the running time of $\max (\mathrm{EFPA}, \mathrm{OPCA})$ is not longer than twice that of EFPA. Given that EFPA is known to be scalable, $\max (\mathrm{EFPA}, \mathrm{OPCA})$ will also be scalable.

The behavior of the convergence of EFPA (which also applies to OPCA) is interesting. We will attempt to provide an intuitive explanation for this behavior. It seems that there are two main factors at play that affect the speed of convergence. One factor is the level of traffic (and its effect on the proportion of overflow traffic) and the other is the sensitivity of the EFPA blocking probability to the load. Consider the $80 \%$ traffic load (40 erlangs) scenario presented in Fig. 3. Notice also the very steep gradient in the region. In such a case, significant error in end-to-end blocking probability prediction possibly implies also significant errors in link blocking probability. This leads, in turn to a significant error in overflow predictions which again cause errors in end-to-end blocking probability which slows down the convergence. On the other, consider for example the heavy traffic scenario represented by the region around $48 \mathrm{er}-$ langs in Fig. 3. In this case, the end-to-end blocking probability is not sensitive to changes in the load and errors in blocking lead to less significant errors in overflow traffic which lead to faster convergence than the previous 40 erlangs scenario. Now consider the 39 erlangs scenario in Fig. 3, where the gradient is at least as steep as in the 40 erlangs scenario. However, in this case, since we have lighter traffic and the overflow load is far less significant (notice the drop in blocking probability by two order of magnitudes in the 39 erlangs relative to the 40 erlangs case), errors in prediction of the overflow will not lead to significant errors in total offered load to the links and therefore

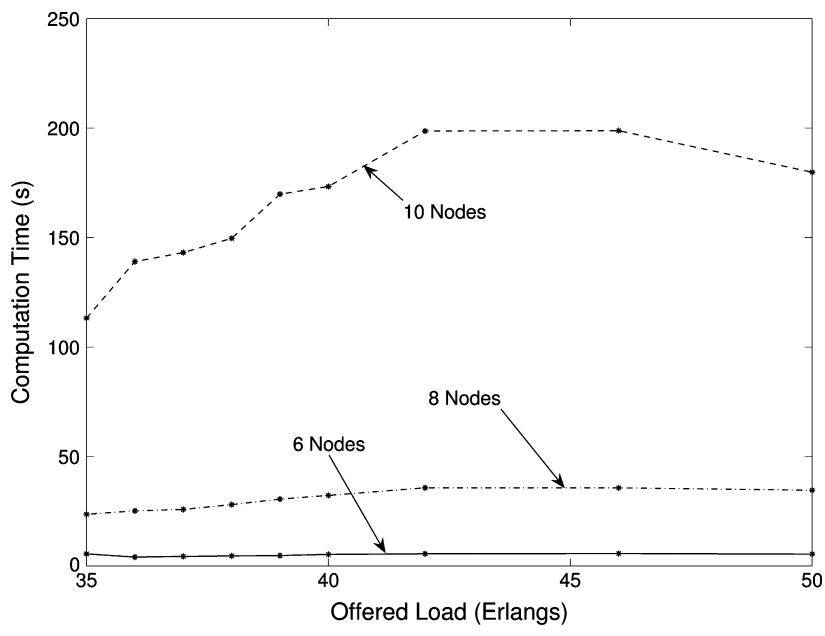

Fig. 16. Running times of OPC for a 6-node, 8-node and 10-node fully meshed topology with $90 \%$ reservation threshold. The maximal number of deflections is 4,6 and 8 for the 6,8 , and 10 node networks, respectively.

we may expect faster convergence than in the 40 erlangs case. Traffic lighter than 39 erlangs where the blocking probability is very small may lead to unexpected behavior of running time due to numerical computation difficulties.

The above is consistent with the peak observed in running time in Fig. 15 at 40 erlangs for the 10-node case. Notice also the less steep gradient in Fig. 2 and much less in Fig. 1 which explains the lower peak for the 8-node case and the almost no peak for the 6-node case. The results presented for OPCA in Fig. 16 around 40 erlangs for the 10-node case are high but not as high as the peak of EFPA presented in Fig. 15. This is consistent with our explanation. Observe that in Fig. 3, the OPCA gradient at 40 is not as steep at that of EFPA.

\section{H. Sensitivity to Burst Length}

Here we examine the sensitivity of $\max (\mathrm{EFPA}, \mathrm{OPCA})$ to burst length. First, we fix the mean of the burst size and examine the sensitivity of $\max (\mathrm{EFPA}, \mathrm{OPCA})$ to higher moments (shape of the distribution) by considering burst length distributions other than exponential, as the exponential distribution assumed by $\max (\mathrm{EFPA}, \mathrm{OPCA})$ may not be the right one to characterize the burst size. Then, we vary the burst mean, maintaining the exponential distribution, to examine the accuracy of max(EFPA, OPCA) for a wide range of mean burst lengths. This will validate the applicability of $\max (\mathrm{EFPA}, \mathrm{OPCA})$ to OPS and OBS. Furthermore, as mentioned above, long bursts may be served by several trunks simultaneously - an effect which is not considered by $\max (\mathrm{EFPA}, \mathrm{OPCA})$, so it is important to know the error this introduces.

For both examinations, we consider a 6-node fully meshed network with 50 links per trunk with $90 \%$ reservation threshold. The number of deflections is limited to 4 .

1) Sensitivity to Shape of the Burst Size Distribution: To study the sensitivity of max (EFPA, OPCA) to higher moments of the burst size distribution we consider the following four scenarios. In the first scenario the burst length is exponentially distributed with mean 1 . In the second scenario, we maintain the mean burst size the same as in the first scenario (equal to 1), but 


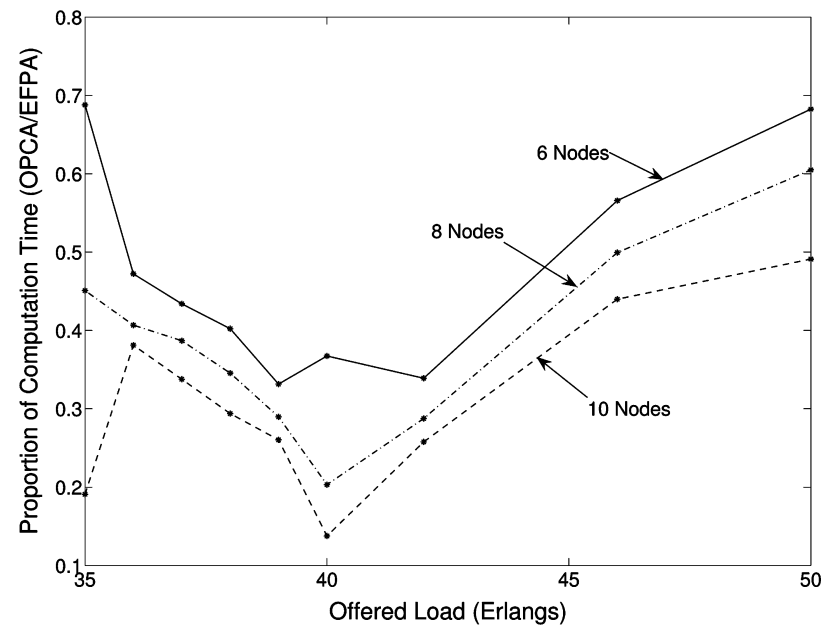

Fig. 17. Ratios of running times OPCA/EFPA for a 6-node, 8-node and 10 -node fully meshed topology with $90 \%$ reservation threshold. The maximal number of deflections is 4,6 and 8 for the 6,8 , and 10 node networks, respectively. A value of 0.5 means that OPCA took half the time.

we increase its variance. In particular, we consider the case that the burst length is hyper exponentially distributed. In particular, with probability $1 / 6$ it is exponentially distributed with mean 5 , and with probability $5 / 6$ it is exponentially distributed with mean $1 / 5$. In the third scenario, we further increase the variance of the burst length but maintain its mean of 1 . We consider the burst length to be hyper exponential distributed: with probability $1 / 11$ it is exponentially distributed with mean 10 , and with probability $10 / 11$ it is exponentially distributed with mean $1 / 10$. In the fourth scenario, we consider a heavy tail distribution for the burst-size maintaining its mean at 1 , but with infinite variance. In particular, we consider the burst size $B$ to follow a Pareto distribution with a complementary distribution function (CDF) that takes the form:

$$
\operatorname{Prob}(B>x)= \begin{cases}\left(\frac{\delta}{x}\right)^{\gamma}, & x \geq \delta, \\ 1, & \text { otherwise },\end{cases}
$$

where $\delta>0$ [seconds] is the scale parameter (minimum burst size) and $\gamma>0$ is the shape parameter of the Pareto distribution. The mean of $B$ is given by

$$
\mathrm{E}(B)= \begin{cases}\infty, & 0<\gamma \leq 1 \\ \frac{\delta \gamma}{\gamma-1}, & \gamma>1\end{cases}
$$

For $0<\gamma \leq 2$, the variance $\operatorname{Var}(B)=\infty$. In our simulation we set $\delta=0.5$ and $\gamma=2$.

Simulation results for the blocking probability are presented in Fig. 18. In these simulation results, the horizontal axis represents the actual (not theoretical) traffic load observed during the simulation. Such presentation of results is important when heavy tailed random deviates are generated because there can be significant difference between the expected and the measured traffic load. We observe that the blocking probability results for the first three scenarios are indistinguishable when plotted - demonstrating very weak sensitivity to the second and higher moments of burst length distribution. The fourth scenario related to heavy tailed bursts gives a slightly different blocking

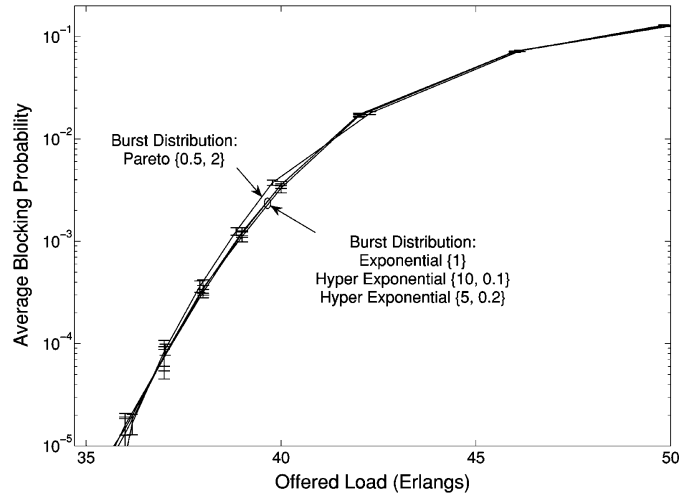

Fig. 18. Blocking probability versus traffic load for four different burst size distributions for a 6 -node, fully meshed network with $90 \%$ reservation threshold. Number of deflections limited to 4 .

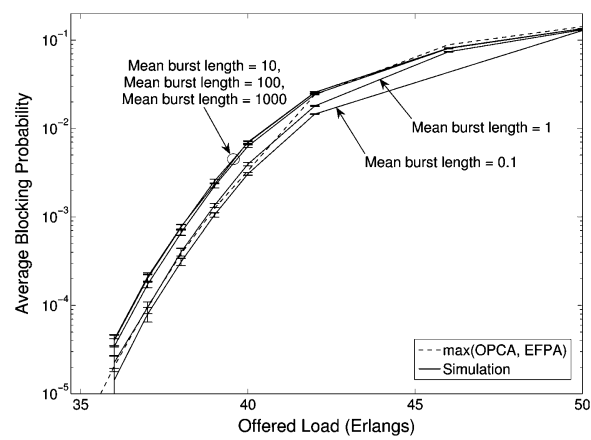

Fig. 19. Blocking probability versus traffic load for four different burst size means for a 6-node, fully meshed network with $90 \%$ reservation threshold. Number of deflections limited to 4 .

probability curve, but still very close to the results of the other three scenarios. This is consistent with the bufferless nature of the network we consider. This is also consistent with the above mentioned result related to the $\mathrm{M} / \mathrm{M} / \mathrm{k} / \mathrm{k}$ system which is known to give blocking probabilities which are insensitive to the form of the burst length distribution and only depends on its mean. This also indicates that the accuracy of $\max (\mathrm{EFPA}, \mathrm{OPCA}$ ) does not depend significantly on the form of the burst length distribution.

2) Sensitivity to the Mean Burst Size: To study the sensitivity of $\max (\mathrm{EFPA}, \mathrm{OPCA})$ to the mean burst size, we consider five cases where the mean burst size is equal to 0.1 , $1,10,100$ and 1000. Notice that all other previous results presented above the mean is equal to 1 . Simulation results and $\max (\mathrm{EFPA}, \mathrm{OPCA})$ approximations for the blocking probabilities in the five cases are presented in Fig. 19. As expected, in the interesting range where the blocking probability is between $10^{-3}$ and $10^{-4}, \max (\mathrm{EFPA}, \mathrm{OPCA})$ underestimate somewhat the blocking probability in the case of larger bursts, probably due to dependency between loads on consecutive trunks created by long bursts which is not considered by $\max (\mathrm{EFPA}, \mathrm{OPCA})$. Nevertheless, the error in dimensioning, namely, the difference in traffic load between the simulation results and $\max (\mathrm{EFPA}, \mathrm{OPCA})$ in the case of $10^{-3}$ blocking probability, for the large bursts scenarios, i.e., mean burst $=100$ or 1000 , is no more that $2 \%$. 


\section{CONCLUSION}

We have demonstrated that $\max (\mathrm{EFPA}, \mathrm{OPCA})$ is a reasonably accurate approach to approximate blocking probability of OBS and OPS networks using deflection routing with sufficient protection to avoid instability. Although in some cases $\max (\mathrm{EFPA}, \mathrm{OPCA})$ is not perfectly accurate, especially in the load region within the gap between the high and low traffic load, $\max (\mathrm{EFPA}, \mathrm{OPCA})$ is clearly an improvement over EFPA and almost always conservative. We have demonstrated that even for the most inaccurate scenario, if we aim to dimension a network using the $\max (\mathrm{EFPA}, \mathrm{OPCA})$ approximation, we will only over-dimension the network by some $2 \%$ which is an acceptable error. We have further demonstrated that the results are not sensitive to the shape of burst size distribution and that for large bursts $\max (\mathrm{EFPA}, \mathrm{OPCA})$ may under-estimate required dimensioning by around $2 \%$. By empirical evidence, the running time of $\max (\mathrm{EFPA}, \mathrm{OPCA})$ is no longer than twice that of EFPA so max(EFPA, OPCA) is computationally efficient and scalable.

We have also demonstrated that in certain topologies where there are many alternative routes, e.g., fully meshed topology, a significant improvement in utilization can be achieved by using multiple deflections. Using our results, we have demonstrated the reduced marginal benefit of increasing the maximal allowable number of deflections and evaluated the achievable utilization as a function of this number.

In topologies where there are not many alternative routes, $\max (\mathrm{EFPA}, \mathrm{OPCA})$ does not improve significantly the already accurate results of EFPA. Nevertheless, max(EFPA, OPCA) is not less accurate than EFPA in such networks, and since it is robust and applicable to a wider range of topologies, $\max (\mathrm{EFPA}, \mathrm{OPCA})$ has an overall advantage over EFPA.

Although having trunk reservation seems to help the accuracy of $\max (\mathrm{EFPA}, \mathrm{OPCA})$, and in the cases studied we generally obtained good agreement with simulations, there may be pathological cases in which max(EFPA, OPCA) may not be accurate.

\section{REFERENCES}

[1] C. Qiao and M. Yoo, "Optical burst switching (OBS) -A new paradigm for an optical internet," J. High Speed Netw., vol. 8, no. 1, pp. 4468-4492, 1999.

[2] S. J. B. Yoo, "Optical packet and burst switching technologies for the future photonic internet," J. Lightw. Technol., vol. 24, no. 12, pp. 4468-4492, Dec. 2006

[3] S.-P. Chung, A. Kashper, and K. W. Ross, "Computing approximate blocking probabilities for large loss networks with state-dependent routing," IEEE/ACM Trans. Netw., vol. 1, pp. 105-115, 1993.

[4] R. Cooper and S. Katz, "Analysis of alternate routing networks with account taken of nonrandomness of overflow traffic," Bell Tel. Lab. Memo. Tech. Rep., 1964.

[5] F. P. Kelly, "Blocking probabilities in large circuit-switched networks," Adv. Appl. Probab., vol. 18, pp. 473-505, 1986.

[6] I. Baldine, G. N. Rouskas, H. G. Perros, and D. Stevenson, "JumpStart: A just-in-time signaling architecture for WDM burst-switched networks," IEEE Commun. Mag., vol. 40, no. 2, pp. 82-89, Feb. 2002.

[7] H. Overby, N. Stol, and M. Nord, "Evaluation of QoS differentiation mechanisms in asynchronous bufferless optical packet-switched networks," IEEE Commun. Mag., vol. 44, no. 8, pp. 52-57, Aug. 2006.

[8] S. Lee, K. Sriram, H. Kim, and J. Song, "Contention-based limited deflection routing protocol in optical burst-switched networks," IEEE J. Sel. Areas Commun., vol. 23, no. 8, pp. 1596-1611, Aug. 2005.
[9] S. Yao, B. Mukherjee, and S. Dixit, "Advances in photonic packet switching: An overview," IEEE Commun. Mag., vol. 38, no. 2, pp. 84-94, 2000

[10] E. W. M. Wong, M. Y. M. Chiu, M. Zukerman, Z. Rosberg, S. Chan, and A. Zalesky, "A novel method for modeling and analysis of distributed video on demand systems," in Proc. IEEE ICC 2005, Seoul, May 2005, pp. 88-92.

[11] E. W. M. Wong, A. Zalesky, Z. Rosberg, and M. Zukerman, "A new method for approximating blocking probability in overflow loss networks," Comput. Netw., vol. 51, no. 11, pp. 2958-2975, 2007.

[12] G. Raskutti, A. Zalesky, E. W. M. Wong, and M. Zukerman, "Enhanced blocking probability evaluation method for circuit-switched trunk reservation networks," IEEE Commun. Lett., vol. 11, no. 6, pp. 543-545, June 2007.

[13] C. F. Hsu, T. L. Liu, and N. F. Huang, "Performance analysis of deflection routing in optical burst-switched networks," in Proc. IEEE INFOCOM, New York, Jun. 2002, vol. 1, pp. 66-73.

[14] H. Tanida, K. Ohmae, Y.-B. Choi, and H. Okada, "An effective BECN/CRN typed deflection routing for QoS guaranteed optical burst switching," in Proc. IEEE GLOBECOM, San Francisco, Dec. 2003, vol. 5, pp. 2601-2606.

[15] C. M. Gauger, M. Köhn, and J. Scharf, "Comparison of contention resolution strategies in OBS network scenarios," in Proc. 6th ICTON, Wroclaw, Poland, Jul. 2004, vol. 1, pp. 18-21.

[16] S. K. Lee, K. Sriram, H. S. Kim, and J. S. Song, "Contention-based limited deflection routing in OBS networks," in Proc. IEEE GLOBECOM, San Francisco, Dec. 2003, vol. 5, pp. 2633-2637.

[17] C. F. Hsu, T. L. Liu, and N. F. Huang, "On the deflection routing in QoS supported optical burst-switched networks," in Proc. IEEE ICC, Apr. 2002, vol. 5, pp. 2786-1790.

[18] C. F. Hsu and T. L. Liu, "On deflection routing in optical burst-switched networks," J. High Speed Netw., vol. 14, no. 4, pp. 341-362, Oct. 2005.

[19] D. X. Y. Chen, H. Wu, and C. Qiao, "On the deflection routing in QoS supported optical burst-switched networks," in Proc. IEEE ICC, Anchorage, Alaska, May 2003, vol. 2, pp. 1355-1359.

[20] Y. Horiuchi and M. Suzuki, "Demonstration of deflection routing based on optical label in an optical burst switching network," in Proc. 28th Euro. Conf. on Optical Commun. (ECOC 2002), Copenhagen, Sep. 2002, vol. 2, pp. 1-2.

[21] C. Cameron, A. Zalesky, and M. Zukerman, "Prioritized deflection routing in optical burst switching networks," IEICE Trans. Commun., vol. E88-B, no. 5, pp. 1861-1867, May 2005.

[22] A. Zalesky, H. L. Vu, Z. Rosberg, E. W. M. Wong, and M. Zukerman, "OBS contention resolution performance," Perform. Eval., vol. 64, no. 4, pp. 357-373, 2007.

[23] A. Zalesky, H. L. Vu, Z. Rosberg, E. W. M. Wong, and M. Zukerman, "Stabilizing deflection routing in optical burst switched networks," IEEE J. Sel. Areas Commun., vol. 25, no. 6, pp. 3-19, Aug. 2007.

[24] E. Ciaramella, G. Contestabile, F. Curti, and A. D'ottavi, "Fast tunable wavelength conversion for all-optical packet switching," IEEE Photon. Technol. Lett., vol. 12, no. 10, pp. 1361-1363, Oct. 2000.

[25] J. Baliga, E. M. W. Wong, and M. Zukerman, "Analysis of bufferless OBS/OPS networks with multiple deflections," IEEE Commun. Lett., to be published.

[26] V. Eramo and M. Listanti, "Packet loss in a bufferless optical WDM switch employing sharedtunable wavelength converters," J. Lightw. Technol., vol. 18, no. 12, pp. 1818-1833, Dec. 2000.

[27] R. S. Tucker, "The role of optics and electronics in high-capacity routers," J. Lightw. Technol., vol. 24, no. 12, pp. 4655-4673, 2006.

[28] L. Kosten, "On the blocking probability of graded multiples," Nachrichtentech. Elek, vol. 14, pp. 5-12, Jan. 1937.

[29] E. Brockmeyer, "The simple overflow problem in the theory of telephone traffic," Teleteknik, vol. 5, pp. 361-374, 1954.

[30] R. Potter, "Explicit formulae for all overflow moments in Kosten and Brockmeyer system with renewal input," Australian Telecommun. Res., vol. 13, pp. 39-49, 1980.

[31] C. Pearce and R. Potter, "Some formulae old and new for overflow traffic in telephony," Australian Telecommun. Res., vol. 11, pp. 92-97, 1977.

[32] A. Kuczura, "The interrupted Poisson process as an overflow process," Bell Syst. Tech. J., vol. 52, no. 3, pp. 437-448, Mar. 1973.

[33] R. Wilkinson, "Theories of toll traffic engineering in the U.S.A.," Bell Syst. Tech. J., vol. 35, no. 2, pp. 421-514, Mar. 1956.

[34] D. Jagerman, "Methods in traffic calculations," AT \& T Bell Lab. Tech. J., vol. 63, no. 7, pp. 1283-1301, Sep. 1984. 
[35] A. Fredericks, "Congestion in blocking systems-A simple approximation technique," Bell Syst. Tech. J., vol. 59, no. 6, pp. 805-827, Jul.-Aug. 1980.

[36] A. Kuczura and D. Bajaj, "A method of moments for the analysis of a switched communication networks performance," IEEE Trans. Commun., vol. COM-25, no. 2, pp. 185-193, Feb. 1977.

[37] A. Girard, Routing and Dimensioning in Circuit-Switched Networks. Boston, MA: Addison-Wesley, 1980.

[38] Q. Huang, K.-T. Ko, and V. B. Iversen, "Approximation of loss calculation for hierarchical networks with multiservice overflows," IEEE Trans. Commun., vol. 56, no. 3, pp. 466-473, Mar. 2008.

[39] R. S. Krupp, "Stabilization of alternate routing networks," in Proc. IEEE ICC, Philadelphia, PA, Jun. 1982, pp. 31.2.1-31.2.5.

[40] D. Bertsekas and R. Gallager, Data Networks. : Prentice Hall, 1992.

[41] L. Kosten, Stochastic Theory of Service Systems. New York: Pergamon Press, 1973

[42] D. Gross, J. F. Shortle, J. M. Thompson, and C. M. Harris, Fundamentals of Queueing Theory. Hoboken, NJ: Wiley, 2008.

[43] S. M. Ross, Stochastic Processes. New York: Wiely, 1996.

[44] L. Kosten, "On the validity of the Erlang and Engset loss formulae," Het PTT-Bedrijf, vol. 2, no. 42, pp. 42-45, 1948.

[45] L. Takacs, "On Erlang's formula," Ann. Math. Statist., vol. 40, pp. 71-78, 1969.

[46] C. Palm, "Analysis of the Erlang traffic formula for busy-signal assignment," Ericsson Technics, vol. 6, pp. 39-58, 1938.

[47] B. A. Sevast'yanov, "An ergodic theorem for Markov processes and its application to telephone systems with refusals," Theory Probab. Appl., vol. 2, no. 1, pp. 104-112, 1957.

[48] X. Yuan, R. Melhem, R. Gupta, Y. Mei, and C. Qiao, "Distributed control protocols for wavelength reservation and their performance evaluation," Photonic Netw. Commun., vol. 1, no. 3, pp. 207-218, Nov. 1999.

[49] J. Li, C. Qiao, and Y. Chen, "Recent progress in the scheduling algorithms in optical-burst-switched networks," J. Opt. Netw., vol. 3, no. 4, pp. 229-241, 2004.

[50] T. Durhuus, B. Mikkelsen, C. Joergensen, S. L. Danielsen, and K. E. Stubkjaer, "All-optical wavelength conversion by semiconductor optical amplifiers," J. Lightw. Technol., vol. 14, no. 6, pp. 942-954, June 1996.

[51] M. Zukerman, E. W. M. Wong, Z. Rosberg, G. M. Lee, and H. L. Vu, "On teletraffic applications to OBS," IEEE Commun. Lett., vol. 8, no. 2, pp. 116-118, Feb. 2004.

[52] C. Qiao, "Labeled optical burst switching for IP-over-WDM integration," IEEE Commun. Mag., vol. 38, no. 9, pp. 104-114, 2000.

[53] L. Battestilli and H. Perros, "A performance study of an optical burst switched network with dynamic simultaneous link possession," Comput. Netw., vol. 50, no. 2, pp. 219-236, Feb. 2006.

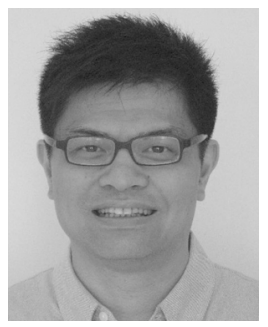

Eric W. M. Wong (S'87-M'90-SM'00) received the B.Sc. and M.Phil. degrees in electronic engineering from the Chinese University of Hong Kong, Hong Kong, in 1988 and 1990, respectively, and the Ph.D. degree in electrical and computer engineering from the University of Massachusetts, Amherst, in 1994.

In 1994, he joined the City University of Hong Kong, where he is now an Associate Professor with the Department of Electronic Engineering. His research interests include the analysis and design of telecommunications networks, optical switching and video-on-demand systems. His most notable research work involved the first accurate and workable model for state-dependent dynamic routing. Since 1991, the model has been used by AT\&T to design and dimension its telephone network that uses real-time network routing.

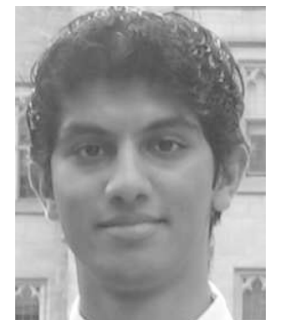

Jayant Baliga received both the B.Sc. degree in computer science and the B.E. degree in electrical and electronic engineering (with first class honors) from the University of Melbourne, Australia, in 2007, where he is currently working toward the Ph.D. degree.

His research interests include optical network architectures and wireless communications.

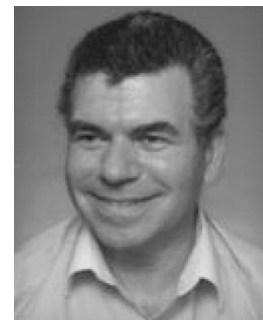

Moshe Zukerman (M'87-SM'91-F'07) received the B.Sc. degree in industrial engineering and management and the M.Sc. degree in operations research from the Technion-Israel Institute of Technology, Haifa, Israel, and the Ph.D. degree in engineering from University of California, Los Angeles, in 1985.

$\mathrm{He}$ was an independent Consultant with the IRI Corporation and a Postdoctoral Fellow with the University of California, Los Angeles, in 1985-1986. In 1986-1997, he was with the Telstra Research Laboratories (TRL), first as a Research Engineer and, in 1988-1997, as a Project Leader. He also taught and supervised graduate students at Monash University in 1990-2001. During 1997-2008, he was with The University of Melbourne, Victoria, Australia. In 2008 he joined City University of Hong Kong as a Chair Professor of Information Engineering, and a team leader He has over 200 publications in scientific journals and conference proceedings.

Dr. Zukerman has served on various editorial boards such as Computer Networks, IEEE Communications Magazine, the IEEE JOURNAL OF SELECTED ARES IN COMMUNICATIONS, IEEE TRANSACTIONS ON NETWORKING and the International Journal of Communication Systems.

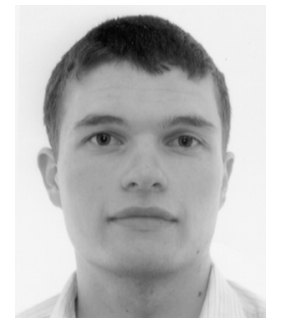

Andrew Zalesky received the B.Sc. degree in 2002 the B.E. degree in electrical engineering in 2003 and the $\mathrm{Ph} . \mathrm{D}$. degree in engineering in 2006, all from the University of Melbourne, Australia.

In 2008, he was an Australian Research Council (ARC) International Fellow. He is currently an ARC Postdoctoral Fellow based at the University of Melbourne. His research interests are in modeling and performance evaluation of natural and engineered systems. Since commencing postdoctoral research, he has received support from the ARC, Australian Academy of Science, American Australian Association and the CASS Foundation.

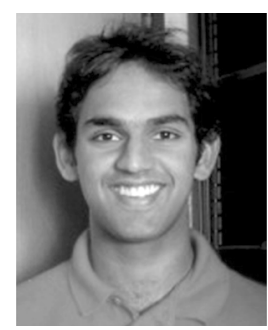

Garvesh Raskutti received the B.Sc. degree in mathematics and the B.E. degree in electrical and electronic engineering, both in 2007, and the M.E.Sc. degree in 2008 from the University of Melbourne, Australia. He is currently undertaking a Ph.D. degree in statistics at the University of California Berkeley.

His research interests include computer networks and statistical machine learning. 